\title{
Experiencia en pacientes operados de roncopatía y apnea obstructiva del sueño
}

\section{Clinical experience in patients with surgery of rhonchopathy and obstructive sleep apnea syndrome}

\author{
Luis Cabezas Labrín', Laura Hernández Rivero² .
}

\begin{abstract}
RESUMEN
Introducción: La uvulopalatofaringoplastía (UPPP) es un procedimiento ampliamente difundido en el tratamiento del ronquido y el síndrome de apnea e hipopnea obstructiva del sueño (SAHOS), ya que permite aumentar el área de sección transversal de la vía aérea superior y eliminar tejidos obstructivos. Conocer el grado de satisfacción de los pacientes con la cirugía es importante para nuestro desempeño.

Objetivo: Evaluar la satisfacción de los pacientes con el procedimiento UPPP con cirugía nasal con datos subjetivos mediante la aplicación de una encuesta para ser respondida de forma anónima.

Material y método: Estudio observacional, descriptivo. Se envió vía email una encuesta de 4 preguntas cerradas creada en la plataforma online MonkeySurvey a los pacientes que fueron sometidos a UPPP (faringoplastía de relocalización) con cirugía nasal entre 2015 y 2016.

Resultados: 27 pacientes respondieron la encuesta. Sesenta y seis coma seis por ciento tenían ronquido primario y/o SAHOS leve, 33,3\% SAHOS severo. La edad media al momento de la cirugía fue 41 años. El tiempo de seguimiento medio fue 10 meses (324 meses). Noventa y dos coma cinco por ciento de los pacientes están satisfechos con la cirugía UPPP; 81,4\% refieren que recomendarían la cirugía a otra persona. Noventa y dos coma cinco por ciento refieren mejoría en los ronquidos. Noventa y cinco coma seis por ciento de los pacientes con somnolencia diurna refiere mejoría.

Conclusión: La cirugía UPPP con técnica faringoplastía de relocalización combinada con cirugía nasal en pacientes con ronquido primario y SAHOS ha demostrado una alta tasa de satisfacción según la percepción de los pacientes en el seguimiento desde los 3 meses hasta los 2 años posoperatorios.
\end{abstract}

Palabras clave: Ronquido, apnea, UVPP.

\begin{abstract}
Introduction: Uvulopalatopharyngoplasty (UPPP) is a widely used surgical procedure for snoring and obstructive sleep apnea syndrome (OSAS), since it allows to increase of the
\end{abstract}

\footnotetext{
1 Departamento de Otorrinolaringología, Clínica Las Condes.

2 Cirugía del Ronquido y Apnea del Sueño, Clínica Las Condes.
}

Los autores declaran no tener conflictos de interés.

Recibido el 29 de marzo, 2017. Aceptado el 1 de febrero, 2019. 
cross-sectional area of the upper airway and elimination of obstructive tissues. Knowing the degree of satisfaction of patients with surgery is important for our performance.

Aim: To evaluate patient satisfaction with UPPP procedure (relocation pharyngoplasty) with nasal surgery with subjective data through the application of a survey to be answered anonymously.

Material and method: Observational, descriptive study. A survey of 4 closed questions created in the MonkeySurvey online platform was sent to patients who underwent UPPP with nasal surgery between 2015 and 2016 via email.

Results: 27 patients answered the survey. $66.6 \%$ had primary snoring and/or mild OSAS, $33.3 \%$ severe OSAS. The average age at the time of surgery was 41 years. The mean followup time was 10 months (3-24 months). 92.5\% of patients are satisfied with UPPP surgery; $81.4 \%$ reported that they would recommend surgery to another person. $92.5 \%$ reported improvement in snoring. $95.6 \%$ of patients with daytime somnolence reported improvement.

Conclusion: UPPP surgery with relocation pharyngoplasty technique combined with nasal surgery in patients with primary snoring and OSAS has shown a high satisfaction rate according to the perception of patients at follow-up from 3 months to 2 years postoperative.

Key words: Ronchopaty, apnea, UPPP.

\section{INTRODUCCIÓN}

Los trastornos respiratorios relacionados con el sueño incluyen un rango de enfermedades que van desde el ronquido primario en un extremo, hasta la apnea obstructiva del sueño en el otro ${ }^{1}$. El ronquido es un sonido, generalmente inspiratorio, consecuencia de la vibración de regiones en la faringe y afecta hasta al $30 \%$ de las mujeres y $50 \%$ de los hombres en edad media ${ }^{1-3}$. El síndrome de apnea e hipopnea obstructiva del sueño (SAHOS) se caracteriza por episodios de obstrucción parcial 0 total de la vía aérea superior durante el sueño y su prevalencia es del 6\%-10\% en hombres y $4 \%$ $6 \%$ en mujeres ${ }^{2,4-6,13}$; su síntoma más frecuente son los ronquidos $(94 \%)^{2,13}$. Ambas entidades tienen un severo impacto en la calidad de vida del individuo, con disminución en la calidad del sueño y somnolencia diurna ${ }^{1-7}$.

El tratamiento quirúrgico para el ronquido y el SAHOS tiene como objetivo aumentar el área de sección transversal de la vía aérea superior y eliminar tejidos obstructivos ${ }^{8}$. Dentro de las alternativas quirúrgicas, la uvulopalatofaringoplastía (UPPP, por sus siglas en inglés) es uno de los procedimientos más ampliamente difundidos, demostrando éxito en la reducción de los síntomas y mejoría en la calidad de vida ${ }^{2,3,9,10}$, ya que la mayoría de estos pacientes cursa con algún grado de estrechamiento de la vía aérea en el nivel velofarínge $0^{9}$. Sin embargo, su utilidad continúa siendo controversial, particularmente en el SAHOS moderado-severo, debido a la falta de estudios controlados y criterios estandarizados para definir su eficacia ${ }^{2,9,10}$, por lo que la aplicación de presión positiva continua en la vía aérea (CPAP) es el tratamiento de elección en esta entidad ${ }^{1-10}$.

Múltiples técnicas quirúrgicas de UPPP han sido descritas, entre ellas la de relocalización, que incluye el avance del paladar blando y la remodelación de la pared lateral faríngea para el aumentar el espacio aéreo y disminuir el colapso faríngeo. Esta técnica ha tenido resultados prometedores manteniendo la normalidad de la función faríngea siendo una alternativa favorable en los pacientes con SAHOS ${ }^{11}$. La cirugía nasal, aunque por sí sola no se considera una terapia de primera elección en el tratamiento del SAHOS, ha demostrado proporcionar mejoría significativa en la calidad de vida en pacientes con desórdenes respiratorios del sueño y es potencialmente útil como parte del enfoque de la cirugía multinivel al modificar alteraciones estructurales presentes; adicionalmente, constituye una intervención de valor en pacientes que necesitarán terapia con CPAP ${ }^{1,12,14}$.

El nivel de satisfacción de los pacientes después de la cirugía de UPPP ha sido evaluado previamente con datos subjetivos por Browaldh y cols (2011) en un estudio de 15 años de seguimiento, en donde concluyen que existe una alta tasa de satisfacción y la mayoría de los pacientes 
consideran haber mejorado sus síntomas ${ }^{13}$. Hicklin y cols $(2000)^{15}$ y Koay y cols $(1995)^{15}$ también realizaron estudios que reflejan una alta tasa de éxito a corto plazo, la cual disminuye con el transcurso de los años. Conocer el grado de satisfacción de los pacientes con los procedimientos quirúrgicos que estamos implementando en la actualidad es importante para nuestro desempeño. Además de medir parámetros objetivos, resulta importante para nosotros estar al tanto de la percepción de los pacientes con los resultados de la cirugía, ya que consideramos que esto es también un reflejo de la atención que estamos brindando y nos permite indagar en las causas de insatisfacción.

\section{OBJETIVO}

El objetivo de este estudio fue evaluar la satisfacción de los pacientes con el procedimiento UPPP (faringoplastía de relocalización) con cirugía nasal mediante la aplicación de una encuesta para ser respondida de forma anónima vía email, por individuos sometidos a cirugía por ronquido simple o SAHOS en 2015 y 2016.

\section{MATERIAL Y MÉTODO}

Se realizó un estudio observacional descriptivo, determinando mediante revisión de fichas clínicas, el número total de pacientes con ronquido simple y SAHOS que fueron sometidos a faringoplastia de relocalización con cirugía nasal (septoplastía, turbinoplastía 0 ambas) entre 2015 y 2016. Mediante la plataforma de internet SurveyMonkey se creó una encuesta que se envió por email a la muestra total de pacientes operados, especificando la finalidad de la misma y garantizando el anonimato de sus datos personales y sus respuestas. Esta encuesta estaba centrada en las siguientes cuatro preguntas cerradas:
Se tabularon las respuestas a cada pregunta observando el porcentaje correspondiente a cada una.

\section{Procedimiento quirúrgico}

Todas las cirugías fueron realizadas por el mismo cirujano, con los pacientes bajo anestesia general con intubación orotraqueal, en posición de Rose, utilizando abrebocas de Mclvor. Conforme al procedimiento de faringoplastía de relocalización (Li y Lee, 2009) se realizó amigdalectomía bilateral y resección de mucosa y de tejido adiposo submucoso del área supratonsilar comprendida entre dos líneas que conectan la base de la úvula y el margen inferior del pilar anterior. Se dividió el músculo palatofaríngeo en su extremo superior y los dos colgajos de pilar posterior resultantes se suturaron reposicionados hacia el músculo palatogloso con suturas estilo colchón de Vycril 2-0. Se repitió el procedimiento del lado opuesto y finalmente se resecó el tercio distal de la úvula. Las septoplastías fueron realizadas mediante incisión caudal e identificación del plano submucopericóndrico septal con extirpación de las porciones desviadas y las turbinoplastías mediante reducción del tejido submucoso con radiofrecuencia y fractura lateral.

\section{RESULTADOS}

La encuesta de satisfacción con la cirugía fue enviada vía email a un total de 47 pacientes roncadores y con diagnóstico de SAHOS sometidos a faringoplastía de relocalización con cirugía nasal entre 2015 y 2016. Se realizaron 3 intentos en un periodo de 1 semana, reenviando la encuesta a los pacientes que no la habían completado. La encuesta fue completada finalmente por 27 pacientes $(57 \%$ del total de cirugías), constituyendo la muestra para

\begin{tabular}{|l|l|}
\hline Preguntas & Posibles respuestas \\
\hline ¿Está usted satisfecho con la cirugía de palatofaringoplastía? & Si/No \\
¿Recomendaría la cirugía palatofaringoplastía a otros? & Si/No/No lo sabe \\
¿Actualmente ronca? & Nunca/A veces/Frecuentemente/ Siempre \\
¿Cómo percibe su somnolencia diurna comparado & Curada/Mejor/Sin cambio/ \\
con antes de la cirugía? & No tenía somnolencia diurna antes de la cirugía \\
\hline
\end{tabular}


este estudio (Figura 1). Proponemos que la pérdida de participantes en esta fase se podría atribuir a falla del registro en los datos de contacto de los pacientes, en este caso mal registro del correo electrónico en las fichas médicas, así como también a indisposición de los pacientes a responder la encuesta por razones que no podríamos objetivar (falta de tiempo, olvido o incluso no se podría descartar descontento con la cirugía).

El número de pacientes roncadores, así como el número de pacientes con SAHOS y la severidad del mismo diagnosticado con polisomnografía se muestra en la Tabla 1.

Consideramos los pacientes a los que no se les indicó polisomnografía como roncadores primarios. La edad media al momento de la cirugía fue 41 años (21-59 años). El tiempo de posoperatorio medio fue 10 meses (3-24 meses). Los porcentajes correspondientes a las respuestas a cada pregunta de la encuesta se resumen en la Figura 2 y Tabla 2.

\section{DISCUSIÓN}

Con los resultados de este estudio, evidenciamos que la mayoría de los pacientes sometidos a UPPP, en este caso faringoplastía de relocalización en combinación con cirugía nasal, están satisfechos con la cirugía $(92,5 \%)$ y la recomendarían a otros $(81,4 \%)$. Asimismo, la satisfacción de los pacientes parece mantenerse estable en el transcurso del tiempo posoperatorio. La mayoría de los pacientes alcanzó también la mejoría de los ronquidos (nunca 0 a veces $-92,5 \%$ ) y somnolencia diurna (curada 0 mejor - 95,6\%).

Estos resultados son comparables a los obtenidos por Browaldh y cols (2011), que concluyen, según los datos subjetivos generados por una encuesta similar, que el $78 \%$ de los pacientes con

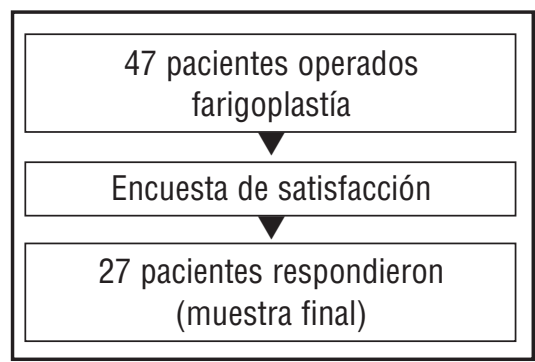

Figura 1. Pacientes operados de faringoplastía que respondieron la encuesta.

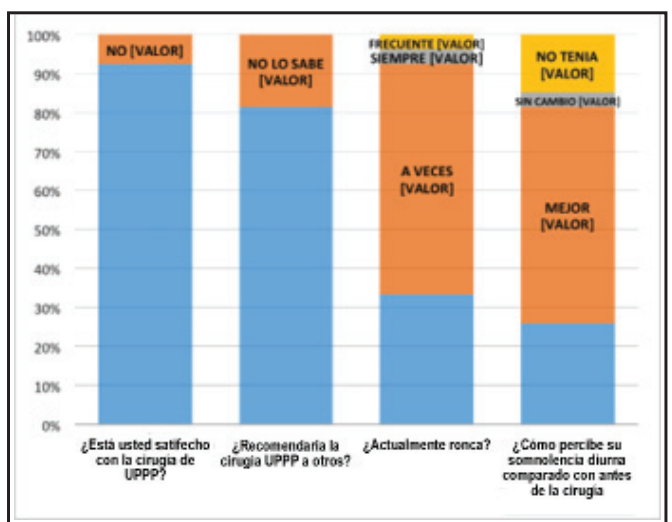

Figura 2. Porcentajes de satisfacción, recomendación de la cirugía, ronquidos y somnolencia diurna posterior a faringoplastía de relocalización $(n=27)$.

SAHOS sometidos a UPPP estaban satisfechos con la cirugía, $40 \%$ habían mejorado los ronquidos y en el $88 \%$ había mejorado o desaparecido la somnolencia diurna; igualmente, refiere que estos resultados no empeoraron durante el tiempo de seguimiento de 15 años. Debemos recordar que al estar estudiando parámetros subjetivos los resultados del cuestionario son muy variables y dependerán incluso de las necesidades y prioridades de

Tabla 1. Clasificación de pacientes con roncopatía

\begin{tabular}{|lcc|}
\hline Clasificación & Número de pacientes & $\%$ \\
\hline SIN polisomnografía & 5 & 18,5 \\
CON polisomnografía & 22 & 81,4 \\
Ronquido primario & 8 & 29,6 \\
SAHOS leve & 5 & 18,5 \\
SAHOS severo & 9 & 33,3 \\
Total & 27 & \\
\hline
\end{tabular}


Tabla 2. Resumen de las respuestas de los pacientes a la encuesta.

\begin{tabular}{|l|l|}
\hline \multicolumn{2}{|l|}{ Distribución de respuestas a la encuesta de satisfacción con la cirugía de faringoplastia de relocalización } \\
\hline Preguntas & Respuestas \\
\hline ¿Está usted satisfecho con la cirugía de palatofaringoplastía? & Si: 25 pacientes $(92,5 \%)$ \\
& No: 2 pacientes $(7,4 \%)$ \\
\hline ¿Recomendaría la cirugía palatofaringoplastía a otros? & Si: 22 pacientes $(81,4 \%)$ \\
& No: 0 \\
& No lo sabe: 5 pacientes $(18,5 \%)$ \\
\hline ¿Actualmente ronca? & Nunca: 9 pacientes $(33,3 \%)$ \\
& A veces: 16 pacientes $(59,2 \%)$ \\
& Frecuentemente: 1 paciente $(3,7 \%)$ \\
Siempre: 1 paciente $(3,7 \%)$
\end{tabular}

cada paciente. De este modo, las causas de falta de satisfacción podrían indagarse más profundamente con otros estudios.

La UPPP ha sido catalogada por muchos autores como el tratamiento de elección en roncadores simples. Inicialmente, se reportaron tasas de éxito del $75 \%-100 \%$. A medida que los estudios han aumentado el tiempo de seguimiento posoperatorio de los pacientes sometidos a UPPP, los porcentajes de éxito se han reducido ${ }^{3}$. Esto se evidencia en el estudio de Hicklin y cols $(2000)^{14}$ en el cual realizaron encuestas retrospectivas a 200 pacientes a los que habían realizado UPPP y concluyeron que, con respecto a la mejoría del ronquido, la tasa de éxito a corto plazo había sido de $76 \%$, pero disminuyó hasta 45\% después de 8-10 años. También concluyen que la tasa de satisfacción con la cirugía también declina con el tiempo, parcialmente como reflejo de la tendencia a retornar del ronquido, pero también asociado al nivel de dolor y/o complicaciones posoperatorias. Koay y cols $(1994)^{15}$ realizan observaciones similares en un estudio de 80 pacientes roncadores, evaluados con cuestionarios, concluyendo que, si bien la UPPP es una cirugía muy eficaz y con alta tasa de satisfacción del paciente, la tasa de recurrencia de los ronquidos fue del $13 \%$, reduciendo la tasa de éxito a corto plazo del $96 \%$ a $83 \%$ después de un periodo mínimo de seguimiento de 12 meses. Como dato interesante, refieren que el parámetro que estuvo relacionado con la recurrencia del ronquido fue el índice de masa corporal, el cual era mayor en dichos pacientes con recurrencia.

La UPPP ha demostrado beneficiar a aquellos pacientes con SAHOS que presentan obstrucción retropalatal, si bien es necesario recordar que el SAHOS es una enfermedad multinivel (nasal, faríngeo e hipofaríngeo) y los resultados quirúrgicos estarán relacionados directamente con la presencia y nivel de afectación de cada uno de estos niveles ${ }^{10}$. Sin embargo, algunos autores hacen mención de que la UPPP es un tratamiento efectivo en casos de SAHOS leve y no demuestra ser más 0 menos efectiva en enfermedad severa, lo que está directamente relacionado con el compromiso multinivel y con la necesidad de definir y seleccionar adecuadamente los casos que más se beneficiarán del procedimiento quirúrgico. Esto puede estar reflejado en nuestros resultados, ya que la proporción de pacientes con ronquido simple y SAHOS leve $(66,6 \%)$ fue mayor y los pacientes que reflejaron insatisfacción con la cirugía pertenecían al grupo de SAHOS severo.

Los estudios que reportan resultados de la cirugía nasal en ronquidos no son controlados ni randomizados en cuanto a la definición del éxito de la cirugía, sin embargo, indican de forma general que la cirugía reduce la intensidad del ronquido, mejora la ventilación nasal, la calidad del sueño y disminuye la somnolencia diurna. En cuanto al SAHOS, la 
cirugía nasal no se considera una terapia de primera elección, su tasa de éxito es menor al 20\%, ya que no produce reducción significativa del índice de apnea-hipopnea (IAH) y por lo tanto no mejora la severidad del SAHOS; no obstante, la reducción de la resistencia nasal mejora significativamente el bienestar del paciente, fatiga diurna, calidad del sueño e incluso puede reducir el número de microdespertares ${ }^{13}$. Estas mismas observaciones en cuanto a los efectos de la cirugía nasal podrían tomar parte en la satisfacción que experimentaron los pacientes con la cirugía en este estudio. De esta forma, resulta entonces interesante conocer el grado de satisfacción de los pacientes con la cirugía

\section{BIBLIOGRAFÍA}

1. Georgalas $C$. The role of the nose in snoring and obstructive sleep apnoea: an update. Eur Arch Otorhinolaryngol 2011; 268: 1365-73.

2. Mannarino M, Di Filippo F, Pirro M. Obstructiva sleep apnea syndrome. Eur J Intern Med 2012; 23: 586-93.

3. Trotter M, D’Souza A, Morgan D. Simple snoring: current practice. J Laryngol Otol2003; 117: 164-8.

4. Azagra E, Espinar E, Barrera J, Llamas J, Solano E. Obstructive sleep apnea syndrome (OSAS) Review of the literature. Med Oral Patol Cir Bucal 2012; 17: 925-9.

5. Park J, Ramar K, Olson E. Updates on definition, consequences and management of obstructive sleep apnea. Mayo Clin Proc 2011; 86: 549-55.

6. Contreras A. Síndrome de apnea obstructiva del sueño. Rev Med Clin Condes 2009; 20: 458-69.

7. Varendh M, Berg S, Andersson M. Longterm follow-up of patients operated with uvulopalatopharyngoplasty from 1985 to 1991. Respiratory Medicine 2012; 106: 1788-93.

8. Franklin Ka, Anttila H, Axelsson S, Gislason T, Maasilta P, Myrre KI, Rehnqvist N. Effects ans side-effects of surgery for snoring and obstructive sleep apnea- a systematic review. Sleep 2009; 32: 27-36. de UPPP en cuanto a la percepción de la mejoría de sus síntomas y corroboramos la necesidad de indagar también en parámetros objetivos para evaluar la efectividad de estos procedimientos.

\section{CONCLUSIÓN}

La cirugía UPPP con técnica faringoplastía de relocalización combinada con cirugía nasal en pacientes con ronquido primario y SAHOS tiene una alta tasa de satisfacción en los pacientes en cuanto a la percepción de la mejoría de sus síntomas, en el seguimiento desde los 3 meses hasta los 2 años posoperatorios.

9. Baradaranfar $M$, Edalatkhah M, Dadgarnia $M$, Atighechi S, Behniafard N, Mirvakili A, Halvani A, Baradaranfar A, Meybodi T. The effect of uvulopalatopharyngoplasty with tonsillectomy in patients with obstructive sleep apnea. Indian J Otolaryngol Head Neck Surg 2014; 67: 29-33.

10. Senior B, Rosenthal L, Lumley A, Gerhardstein R, Day R. Efficacy of uvulopalatopharyngoplasty in unselected patients with mild obstructive sleep apnea. Otolaryngol Head Neck Surg 2000; 123: 179-82.

11. Hsuen-Yu Li, LI-Ang LeE. Relocation pharyngoplasty for obstructive sleep apnea. Laryngoscope 2009; 119: 2472-7.

12. Verse T, Maurer J, Pirsig W. Effect of nasal surgery on sleep-related breathing disorders. Laryngoscope 2002; 112: 64-8.

13. Browaldh N, Friberg D, Svanbog E, Nerfeldt P. 15-year efficacy of uvulopalatopharyngoplasty base don objective and subjective data. Acta Oto-Laryngologica 2011; 131: 1303-10.

14. Hormann K, Verse T. Surgery for sleep disordered breathing. 2d ed. Springer, 2010.

15. HickLin L, ToRTeVIn P, Dasan S. Retrospective survey of long-term results and patient satisfaction with uvulopalatopharyngoplasty for snoring. $J$ Laryngol Otol 2000; 114: 675-81.

16. Koay CB, Freeland AP, Stradling JR. Short-and longterm outcomes of uvulopalatopharyngoplasty for snoring. Clin Otolaryngol Allied Sci 1995; 20: 45-8.

\footnotetext{
Correspondencia: Luis Cabezas

Departamento de Otorrinolaringología, Clínica Las Condes. Santiago, Chile.

E mail: Icabezas@clinicalascondes.cl
} 